\title{
Erratum to: The NMR solution structures of the five constituent cold-shock domains (CSD) of the human UNR (upstream of N-ras) protein
}

\author{
Alexander K. Goroncy $\cdot$ Seizo Koshiba $\cdot$ Naoya Tochio $\cdot$ Tadashi Tomizawa \\ Makoto Inoue $\cdot$ Satoru Watanabe $\cdot$ Takushi Harada $\cdot$ Akiko Tanaka \\ Osamu Ohara $\cdot$ Takanori Kigawa $\cdot$ Shigeyuki Yokoyama
}

Published online: 31 March 2010

(C) Springer Science+Business Media B.V. 2010

Erratum to: J Struct Funct Genomics (2010)

DOI 10.1007/s10969-010-9081-z

Unfortunately the fifth author's name had been misspelled. The correct version is Makoto Inoue and not Makato Inoue as published. The authors would like to apologize for this error.

The online version of the original article can be found under doi:10.1007/s10969-010-9081-z.

A. K. Goroncy · S. Koshiba - N. Tochio - T. Tomizawa .

M. Inoue - S. Watanabe - T. Harada - A. Tanaka - T. Kigawa

S. Yokoyama $(\bowtie)$

RIKEN Systems and Structural Biology Center,

1-7-22 Suehiro-cho, Tsurumi-ku, Yokohama,

Kanagawa 230-0045, Japan

e-mail: yokoyama@biochem.s.u-tokyo.ac.jp

O. Ohara

Kazusa DNA Research Institute, Department of Human

Genome Research, 2-6-7 Kazusa-kamatari, Kisarazu,

Chiba 292-0818, Japan

O. Ohara

RIKEN Research Center for Allergy and Immunology,

Laboratory for Immunogenomics, 1-7-22 Suehiro-cho,

Tsurumi-ku, Yokohama, Kanagawa 230-0045, Japan

T. Kigawa

Department of Computational Intelligence and Systems Science, Interdisciplinary Graduate School of Science and Engineering,

Tokyo Institute of Technology, Yokohama,

Kanagawa 226-8502, Japan

S. Yokoyama

Department of Biophysics and Biochemistry, Graduate School of Science, The University of Tokyo, Tokyo 113-0033, Japan 